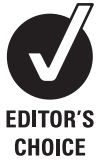

${ }^{1}$ Department of Medicine, Cardiovascular Imaging Center, Cardiac and Vascular Center, Samsung Medical Center, Sungkyunkwan University School of Medicine, Seoul, Korea; ${ }^{2}$ Department of Emergency Medicine, Cardiovascular Imaging Center Cardiac and Vascular Center, Samsung Medical Center, Sungkyunkwan University School of Medicine, Seoul, Korea; ${ }^{3}$ Department of Cardiology, Hanmaeum General Hospital, Jeju, Korea; ${ }^{4}$ Division of Cardiovascular Diseases, Mayo Clinic College of Medicine, Rochester, Minnesota, USA

Correspondence to: Dr Eun-Seok Jeon, Department of Medicine, Cardiovascular Imaging Center, Cardiac and Vascular Center, Samsung Medical Center, Sungkyunkwan University School of Medicine 50 Irwon-dong, Gangnam-ku, Seoul, Korea, 135-710; esjeon@skku.edu

Accepted 14 October 2009 Published Online First 26 October 2009

\title{
Preoperative NT-proBNP and CRP predict perioperative major cardiovascular events in non-cardiac surgery
}

\author{
J-H Choi, ${ }^{1,2}$ D K Cho, ${ }^{3}$ Y-B Song, ${ }^{1}$ J-Y Hahn, ${ }^{1}$ S Choi, ${ }^{1}$ H-C Gwon, ${ }^{1}$ D-K Kim, ${ }^{1}$ S H Lee, ${ }^{1}$ \\ J K Oh, ${ }^{1,4}$ E-S Jeon ${ }^{1}$
}

\begin{abstract}
Objective: To investigate whether simple and noninvasive measurement of $\mathrm{N}$-terminal pro-brain natriuretic peptide (NT-proBNP) and/or C-reactive protein (CRP) can predict perioperative major cardiovascular event (PMCE). Design: Prospective, single-centre, cohort study. Setting: A 1900-bed tertiary-care university hospital in Seoul, Korea

Design and patients: The predictive power of NTproBNP, CRP and Revised Cardiac Risk Index (RCRI) for the risk of PMCE (myocardial infarction, pulmonary oedema or cardiovascular death) were evaluated from a prospective cohort of 2054 elective major non-cardiac surgery patients. Optimal cut-off values were derived from receiver operating characteristic curve (ROC) analysis.

Main outcome measurement: PMCE (myocardial
\end{abstract} infarction, pulmonary oedema or cardiovascular death) within postoperative 30 days.

Results: PMCE developed in a total of 290 patients (14.1\%). Each increasing quartile of NT-proBNP or CRP level was associated with a greater risk of PMCE after adjustment for traditional clinical risk factors. The relative risk (RR) of highest versus lowest quartile was 5.2 for NTproBNP $(p<0.001)$ and 3.7 for CRP $(p<0.001)$. Both NTproBNP (cut-off $=301 \mathrm{ng} /$ ) and CRP (cut-off $=3.4 \mathrm{mg} /$ ) predicted PMCE better than RCRI (cut-off $=2$ ) by ROC analysis $(p<0.001)$. Moreover, the predictive power of RCRI (adjusted RR $=1.5$ ) could be improved significantly by addition of CRP and NT-proBNP to RCRI (adjusted RR 4.6) $(p<0.001)$.

Conclusions: High preoperative NT-proBNP or CRP is a strong and independent predictor of perioperative major cardiovascular event in non-cardiac surgery. The predictive power of current clinical risk evaluation system would be strengthened by these biomarkers.

Perioperative major cardiovascular events (PMCE) such as acute myocardial infarction, pulmonary oedema or primary cardiovascular death are important causes of morbidity in patients undergoing a major non-cardiac surgery. ${ }^{1}$ A number of clinical risk indices using scoring system have been developed, but the predictive power is still insufficient. $^{2-5}$ Moreover, the results of preoperative myocardial stress test were not consistently predictive of risk. ${ }^{6-8} \mathrm{~A}$ simple and strongly predictive non-invasive test is clinically warranted.

We hypothesised that the pathophysiology of cardiovascular disease including inflammation, myocardial ischaemia or increased ventricular filling pressures would be important in the development of PMCE. Then cardiovascular biomarkers reflecting this pathophysiology would be useful for the prediction of perioperative risk. ${ }^{9}$ Based on abundant clinical data, practical availability and background pathophysiology, ${ }^{10-12}$ we reasoned that $\mathrm{N}$-terminal pro-brain natriuretic peptide (NTproBNP) and C-reactive protein (CRP), representative biomarkers of haemodynamic stress and inflammation, respectively, would be predictive of PMCE. We investigated the predictive power of preoperative NT-proBNP and CRP and compared it with a well-validated clinical risk index for perioperative cardiovascular risk in a large prospective cohort of patients undergoing elective major non-cardiac surgery.

\section{METHODS}

\section{Patients}

We enrolled patients who were referred to consulting cardiology physician for the evaluation of preoperative cardiovascular risk if the following criteria were fulfilled; (1) candidates for elective major non-cardiac surgery and aged more than 21 years, and (2) at least one of cardiovascular risk factors such as hypertension, diabetes, angina, history of revascularisation, heart failure or stroke, or (3) abnormal preoperative electrocardiography with pathological $\mathrm{Q}$ wave or non-sinus rhythm. Major non-cardiac surgery was defined by procedures performed in the operating room requiring general, spinal or epidural anaesthesia, after exclusion of very low-risk surgeries such as dermatological, ophthalmological, nasal or auditory procedures.

We prospectively enrolled 2304 consecutive patients from November 2004 to April 2008. The following patients were excluded; surgery was not done within 2 weeks $(n=118)$, significant myocardial ischaemia or those who required open heart surgery $(n=29)$. To avoid bias in the NT-proBNP results from renal insufficiency, 103 patients with preoperative serum creatinine $\geqslant 2.0 \mathrm{mg} / \mathrm{dl}$ $(\geqslant 176.8 \mu \mathrm{mol} / \mathrm{l})$ were also excluded. ${ }^{13}$ The remaining 2054 patients had undergone non-cardiac surgery within 2 weeks and constituted the study cohort (fig 1).

\section{Data collection}

Clinical perioperative cardiovascular risk was assessed according to the Revised Cardiac Risk Index (RCRI) modified by Lee, a well-validated and widely used risk prediction index. ${ }^{145814}$ Briefly, RCRI calculates perioperative risk by sum of 
Figure 1 Study flowchart.

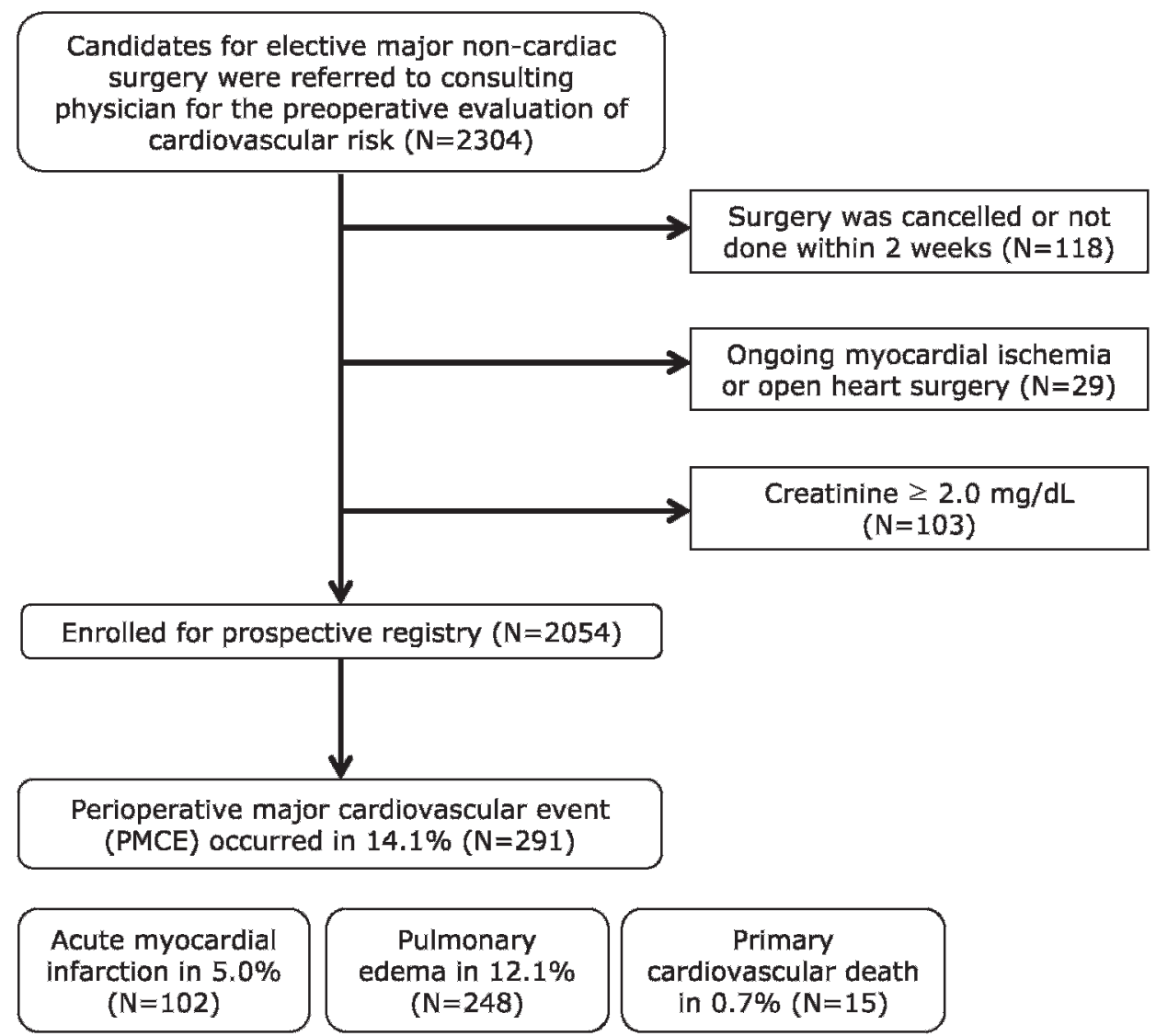

points. Each risk factor, including high-risk surgical procedures, history of ischaemic heart disease, pulmonary oedema, cerebrovascular disease, insulin-dependent diabetes and serum creatinine $>2.0 \mathrm{mg} / \mathrm{dl}$, is assigned one point. The risk of major cardiac event including myocardial infarction, pulmonary oedema, primary cardiac arrest and complete heart block predicted by RCRI was known to be $0.4 \%$ to $11 \%$ according to an RCRI score of 0 to $\geqslant 3$.

The patient's clinical history and functional capacity were evaluated according to the ACC/AHA guidelines on perioperative cardiovascular evaluation and care for non-cardiac surgery. ${ }^{8}$ Basic laboratory tests including electrocardiography, chest x-ray, NT-proBNP and CRP were evaluated within 2 weeks before surgery. Additional non-invasive tests were performed at the physician's discretion. Electrocardiography and serum troponin I were evaluated at the end of the day of surgery and 24 hours later. A chest x-ray was taken on the next day. Any abnormal signs or symptoms suggesting pulmonary oedema or myocardial ischaemia were followed by meticulous evaluation of perioperative cardiac status with repeated cardiac serum markers and electrocardiography. If active pulmonary oedema or ongoing myocardial ischaemia was found, the patient was transferred to the cardiovascular team and treated appropriately. Patients were followed up by the consulting physician until discharge or up to 30 days in hospital after surgery. In case of mortality, the cause of death was decided on the consensus of the surgeon, anaesthesiologist and cardiovascular consulting physician.

The primary endpoint was a perioperative major cardiovascular event (PMCE), which was defined by any single or combined event of secondary endpoints including myocardial infarction, development of pulmonary oedema or primary cardiovascular death. Myocardial infarction was defined by a rise in postoperative troponin I above the 99th percentile of the upper reference limit $(0.78 \mathrm{ng} / \mathrm{ml}$, Roche Diagnostics, Switzerland). Diagnosis of pulmonary oedema required a formal reading of chest $\mathrm{x}$-ray by a radiologist consistent with the complication. Primary cardiovascular death was defined by sudden death that could not be explained by any other noncardiovascular postoperative complications. All clinical events were collected by a research nurse and investigated by physicians. This study protocol was approved by the institutional review board of Samsung Medical Center.

\section{Statistics}

Perioperative risk predictors (RCRI, CRP and NT-proBNP) were treated as continuous variables or ordered categorical variables. Logarithmically transformed values of NT-proBNP and CRP were also used to minimise distribution skewness and kurtosis. The dose-response relation between risk predictors and clinical outcome was investigated by the Jonckheere-Terpstra test for trend. The predictive power of each risk predictor was also quantitatively evaluated with relative risk per $1 \mathrm{SD}$ increase of RCRI and logarithmically transformed biomarker levels. Receiver-operating characteristic (ROC) analysis was performed to calculate sensitivity, specificity, area under the curve and the optimal cut-off. The predictive power of each method was compared using the Hanley and McNail method. ${ }^{15}$ Relative risk was calculated by Zhang and Yu's method. ${ }^{16}$ Relative risk was also calculated for the risk predictors with values categorised by optimal cut-off levels, or a combination of these cut-off levels in an additive manner. Independent predictors of PMCE in univariate analysis were included in multivariate logistic models 
Table 1 Baseline clinical characteristics

\begin{tabular}{|c|c|}
\hline & $\begin{array}{l}\text { Frequency }(\%) \text { or median } \\
\text { (interquartile range) }\end{array}$ \\
\hline Age (years) & $68(61-73)$ \\
\hline Male gender & $1247(60.7)$ \\
\hline Functional class III or IV & $112(5.5)$ \\
\hline Diabetes & $355(17.3)$ \\
\hline Diabetes treated with insulin & $71(3.5)$ \\
\hline Hypertension & $1247(60.7)$ \\
\hline Previous or current heart failure & $62(3.0)$ \\
\hline Previous stroke & $188(9.3)$ \\
\hline Angina & $270(13.2)$ \\
\hline Previous myocardial infarction & $165(8.0)$ \\
\hline Previous revascularisation* & $304(14.8)$ \\
\hline Creatinine $(\mathrm{mg} / \mathrm{dl})$ & $0.9(0.7-1.1)$ \\
\hline NT-proBNP (ng/l) & $109.3(47.0-352.8)$ \\
\hline C-reactive protein $(\mathrm{mg} / \mathrm{l})$ & $2.0(0.7-8.0)$ \\
\hline Electrocardiography & $2054(100)$ \\
\hline Pathological 0 waves & $111(5.4)$ \\
\hline Atrial fibrillation & $164(8.0)$ \\
\hline Left bundle branch block & $14(0.7)$ \\
\hline Echocardiography & $1923(93.6)$ \\
\hline Left ventricular ejection fraction $\leqslant 40 \%$ & $95(4.6)$ \\
\hline Abnormal left ventricular wall motion & $408(19.9)$ \\
\hline Preoperative non-invasive test $\dagger$ & $765(37.2)$ \\
\hline Overall positive result for ischaemia & $188(9.2)$ \\
\hline Preoperative Invasive test & $544(26.5)$ \\
\hline Significant coronary artery disease by invasive test & $359(17.5)$ \\
\hline Any evidence of myocardial ischaemia§ & $444(21.6)$ \\
\hline
\end{tabular}

Data are shown as frequency (\%) or median (interquartile range) as appropriate. *Previous revascularisation includes percutaneous coronary intervention in 222 cases and bypass surgery in 82 cases.

†Preoperative non-invasive test includes SPECT in 577 cases, Treadmill test in 113 cases and stress echocardiography in 47 cases.

$\S P$ sitive non-invasive test or coronary artery stenosis of more than $50 \%$ was defined as any evidence of myocardial ischaemia.

with forward conditional methods. A p value $<0.05$ (two-sided) was considered significant. SPSS version 13.0 was used mostly. ROC curves were compared using Medcalc version 9.6.

\section{RESULTS}

\section{Clinical characteristics}

Preoperative clinical characteristics of the study population are shown in table 1. Briefly, most patients had good functional status without overt heart failure (functional class I or II in $94.6 \%$ and no heart failure in $97.0 \%$ ). A history of angina was found in $13.2 \%$, including $8.0 \%$ of myocardial infarction. Percutaneous coronary intervention (PCI) or coronary artery bypass surgery (CABG) had been performed before non-cardiac surgery in $14.8 \%$. Two-dimensional echocardiography was performed in most patients $(93.6 \%)$, revealing abnormal left ventricular wall motion in $19.9 \%$ of patients. Evidence of myocardial ischaemia which was defined by positive noninvasive test or significant coronary artery stenosis was found in $21.6 \%$. A preoperative $\beta$-blocker or statin was used in $17.3 \%$ and $14.8 \%$, respectively.

\section{Perioperative clinical evaluation}

Most patients received general anaesthesia (97.1\%). Ninetythree patients $(4.5 \%)$ underwent urgent surgery within 24 hours after consultation because of altered clinical situation. These cases were not excluded from the analysis (table 2).
Table 2 Surgical procedure and perioperative risk

\begin{tabular}{|c|c|}
\hline & Frequency $(\%)$ \\
\hline Vascular surgery & $531(25.9)$ \\
\hline Aorta & $160(7.8)$ \\
\hline Suprainguinal vascular & $98(4.8)$ \\
\hline Infrainguinal vascular & $158(7.7)$ \\
\hline Carotid endarterectomy & $97(4.7)$ \\
\hline Other vascular & $18(0.9)$ \\
\hline Non-vascular surgery & $1520(74.4)$ \\
\hline Thorax & $85(4.1)$ \\
\hline Abdomen & $501(24.4)$ \\
\hline Head and neck & $178(8.7)$ \\
\hline Orthopaedic & $439(21.4)$ \\
\hline Prostate & $82(4.0)$ \\
\hline Neurosurgery & $55(2.7)$ \\
\hline Other surgery & $182(8.9)$ \\
\hline General anaesthesia & $1994(97.1)$ \\
\hline Urgent surgery & $93(4.5)$ \\
\hline RCRI (median, interquartile range) & $1(0-2)$ \\
\hline $\mathrm{RCRI}=0$ & $555(27.0)$ \\
\hline $\mathrm{RCRI}=1$ & $846(41.2)$ \\
\hline $\mathrm{RCRI}=2$ & $579(28.2)$ \\
\hline $\mathrm{RCRI}=3$ & $69(3.4)$ \\
\hline $\mathrm{RCRI}=4$ & $5(0.2)$ \\
\hline High risk surgery by $\mathrm{RCRI}{ }^{*}$ & $844(41.1)$ \\
\hline
\end{tabular}

Data are shown as frequency (\%).

*Defined by intraperitoneal, intrathoracic or suprainguinal vascular surgery according to RCRI (Revised Cardiac Risk Index modified by Lee).

\section{Clinical outcomes}

Perioperative major cardiovascular event (PMCE) had developed in 290 (14.1\%) patients. Individual patients may have had more than one event, and all events were counted as an incidence. There were 102 (5.0\%) acute myocardial infarction, 248 (12.1\%) pulmonary oedema and $20(1.0 \%)$ deaths, which were similar to previous studies evaluated patients with risk of cardiovascular disease. ${ }^{1}$ Five patients died because of postoperative disease progression or surgical complication $(0.2 \%)$, and 15 deaths were determined to be primary cardiovascular death (0.7\%), which included three $(0.2 \%)$ acute myocardial infarction, two $(0.1 \%)$ stress-induced cardiomyopathy, ${ }^{17}$ four $(0.2 \%)$ aortic aneurysm rupture or dissection, one $(0.1 \%)$ stroke and five $(0.2 \%)$ sudden death of unknown cause (fig 1) (table 3).

\section{Predictive power of perioperative risk predictors}

We evaluated the continuous values of three risk predictors against perioperative events. Not only increasing score of a clinical predictor, RCRI, but also increasing quartile of biomarker levels, NT-proBNP and CRP, was associated with a greater risk of PMCE $(\mathrm{p}<0.001)$ (fig 2$)$.

Next we investigated whether the risk predictors are related to clinical outcomes independently each other. Each 1-SD increase in RCRI (1 to 2) (adjusted relative risk (RR) $=1.26$ (95\% CI 1.10 to 1.44$)$ ), log CRP (2.7 to $15.1 \mathrm{mg} / \mathrm{l}$ ) (1.74 (1.55 to 1.95)) or log NT-proBNP (135 to $601 \mathrm{ng} / \mathrm{l}$ ) (2.17 (1.91 to 2.45$)$ ) was associated with 1.3-fold to 2.2-fold increased rate of PMCE, even after adjustment for other risk predictors and traditional clinical risk factors. By subgroup analysis, CRP and NT-proBNP were also significantly associated with all secondary endpoints, whereas RCRI was not associated with AMI or primary cardiovascular death. Each 1-SD increase in log CRP or log NT-proBNP was also associated with 1.6-fold to 2.3-fold increased risk of PMCE (table 4). 
Table 3 Clinical outcome

\begin{tabular}{lc}
\hline & Frequency (\%) \\
\hline Perioperative major cardiovascular event (PMCE) & $290(14.1)$ \\
Acute myocardial infarction & $102(5.0)$ \\
$\quad$ Revascularisation & $26(1.3)$ \\
$\quad$ Percutaneous coronary intervention & $24(1.2)$ \\
$\quad$ Coronary artery bypass surgery & $2(0.1)$ \\
New or aggravated heart failure & $248(12.1)$ \\
Primary cardiovascular death* & $15(0.7)$ \\
Acute myocardial infarction & $3(0.2)$ \\
Stress induced cardiomyopathy & $2(0.1)$ \\
Aortic disease & $4(0.2)$ \\
Stroke & $1(0.1)$ \\
Unknown & $5(0.2)$ \\
Death due to postoperative complication or disease progression & $5(0.2)$ \\
All death & $20(1.0)$
\end{tabular}

Data are shown as frequency $(\%)$.

*Death that was not caused by postoperative complication or underlying noncardiovascular disease progression.

\section{Augmentation of predictive power of clinical risk index by addition of biomarkers}

Risk predictors categorised by optimal cut-off levels were used to test whether the predictive power could be increased by the combination of multiple risk factors. RCRI cut-off $(\geqslant 2)$ was associated with 1.5-fold increased risk of PMCE after adjustment for age, sex, and traditional clinical risk factors (adjusted $\mathrm{RR}=1.50$ (95\% CI 1.17 to 1.91)). The risk of PMCE based on CRP cut-off ( $\geqslant 3.4 \mathrm{mg} / \mathrm{l})$ and NT-proBNP cut-off ( $\geqslant 301 \mathrm{ng} / \mathrm{l}$ ) were much higher, 2.7-fold and 3.9-fold, respectively (CRP cutoff, 2.75 (2.16 to 3.45); NT-proBNP cut-off, 3.89 (3.15 to 4.14)) (table 4). Higher CRP and NT-proBNP values were also associated with 2.5-fold to 5.4-fold increased risk of secondary endpoints including AMI, pulmonary oedema, and primary cardiovascular death $(\mathrm{p}<0.05)$, whereas higher RCRI was not associated with AMI or primary cardiovascular death (table 4).

The addition of biomarkers to RCRI increased the relative risk of RCRI for clinical events threefold. For PMCE, the adjusted RR of RCRI increased from 1.50 (95\% CI 1.17 to 1.91) to 4.55 (3.69 to 5.52) after addition of CRP and NT-proBNP. The relative risks for secondary endpoints including AMI, pulmonary oedema and primary cardiovascular death also increased threefold to sevenfold (table 4). The increase of predictive power of RCRI by addition of biomarkers to RCRI was again calculated using ROC analysis. For PMCE, AUC (area under curve) of combination of RCRI cut-off $(\geqslant 2)$ and NT-proBNP cut-off ( $\geqslant 301 \mathrm{ng} / \mathrm{l}), 0.735$ (0.714 to 0.754), and AUC of combination of RCRI cut-off and CRP cut-off $(\geqslant 3.4 \mathrm{mg} / \mathrm{l})$, 0.694 (0.673 to 0.715), were significantly higher than AUC of RCRI cut-off, 0.592 (0.570 to 0.615), ( $p<0.001$, each). AUC of combination of RCRI cut-off and NT-proBNP cut-off and CRP cut-off, 0.772 (0.752 to 0.790), was even much higher than AUC of RCRI cut-off $(p<0.001)$ (fig 3A). The increase in AUC by addition of biomarker cut-offs to RCRI cut-off was also evident in all secondary endpoints including AMI, pulmonary oedema and primary cardiovascular death (fig 3B-D).

\section{DISCUSSION}

Our study showed that the predictive power of a current perioperative clinical risk index could be strengthened significantly by the simple addition of the cardiovascular biomarkers, NT-proBNP and CRP. Our findings can be summarised that the average sensitivity of predicting perioperative major cardiovascular event increased from $59 \%$ to $77 \%$ after addition of biomarkers to clinical risk prediction system.

\section{Predictive power of biomarker versus clinical risk index}

The Revised Cardiac Risk Index modified by Lee (RCRI), which has been shown to be superior to other perioperative risk indices
Figure 2 Clinical outcomes according to the risk predictors. AMI, acute myocardial infarction; CV death, primary cardiovascular death; PMCE, perioperative major cardiovascular event; $\mathrm{PE}$, pulmonary oedema. ${ }^{*} \mathrm{p}<0.001$ by Jonckheere-Terpstra test for trend.
A

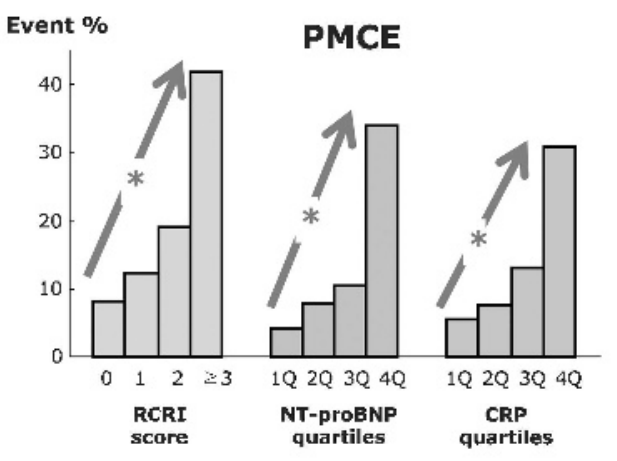

C

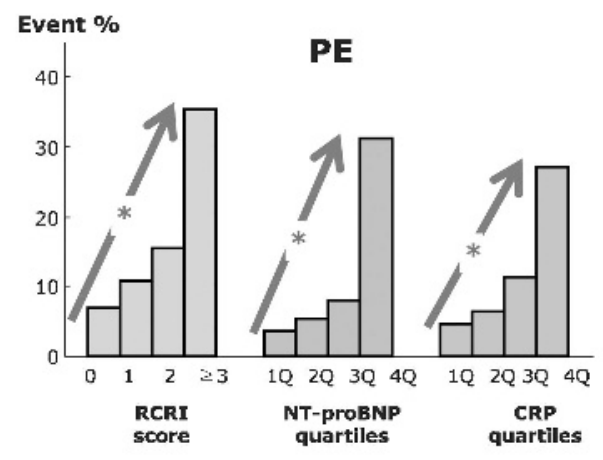

B

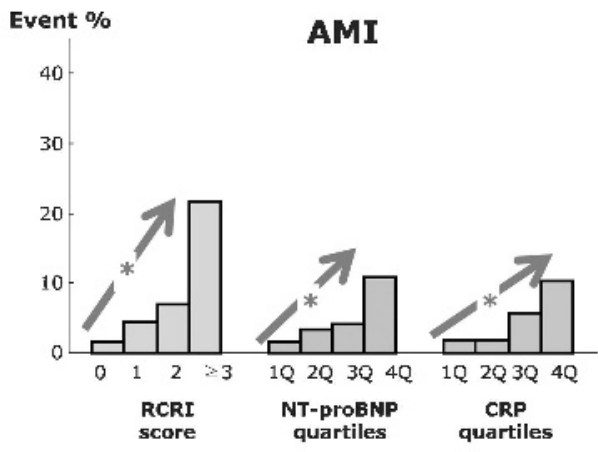

D

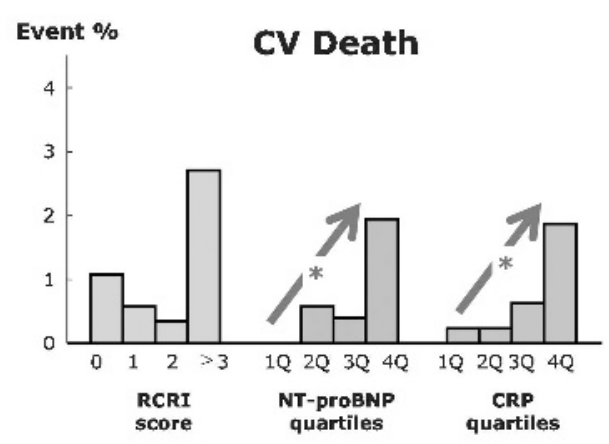


Table 4 Clinical outcomes according to the risk predictors

\begin{tabular}{|c|c|c|c|c|c|c|c|c|}
\hline & \multicolumn{2}{|l|}{ PMCE } & \multicolumn{2}{|l|}{ AMI } & \multicolumn{2}{|l|}{ PE } & \multicolumn{2}{|l|}{ CV death } \\
\hline & RR $(95 \%$ Cl) & p Value & RR $(95 \%$ Cl) & p Value & RR $(95 \%$ Cl) & p Value & RR $(95 \%$ Cl) & p Value \\
\hline \multicolumn{9}{|l|}{ Per 1-SD increase* } \\
\hline RCRI & 1.26 (1.10 to 1.44$)$ & 0.001 & $1.18(0.88$ to 1.56$)$ & 0.27 & 1.30 (1.13 to 1.49$)$ & $<0.001$ & $0.53(0.29$ to 1.00$)$ & 0.05 \\
\hline NT-proBNP & 2.17 (1.91 to 2.45$)$ & $<0.001$ & 1.55 (1.28 to 1.88$)$ & $<0.001$ & 2.27 (1.97 to 2.62 ) & $<0.001$ & 2.30 (1.48 to 3.56$)$ & $<0.001$ \\
\hline \multicolumn{9}{|c|}{ Optimal cut-off of each risk predictors $\dagger$} \\
\hline NT-proBNP & 3.89 (3.15 to 4.74$)$ & $<0.001$ & 2.54 (1.68 to 3.79 ) & $<0.001$ & 4.72 (3.72 to 5.89$)$ & $<0.001$ & $5.39(1.86$ to 15.30$)$ & 0.002 \\
\hline \multicolumn{9}{|c|}{ Combination of best cut-off of each predictors $\S$} \\
\hline $\begin{array}{l}\text { RCRI or CRP or NT- } \\
\text { proBNP } \geqslant \text { cut-off§ }\end{array}$ & 4.55 (3.69 to 5.52$)$ & $<0.001$ & 3.24 (2.10 to 4.92$)$ & $<0.001$ & 5.64 (4.49 to 6.96$)$ & $<0.001$ & 7.71 (2.48 to 23.32$)$ & $<0.001$ \\
\hline
\end{tabular}

Investigate independent association of each risk predictors with clinical outcomes were shown as adjusted relative risk (RR) with 95\% confidence intervals (CI), all three risk predictors were included in the logistic regression analysis with forward conditional method. Analysis was adjusted with all significant univariate risk factors including age and sex. ${ }^{*}$ Measured in linear values, 1-SD increase from mean corresponded to 1 to 2 for RCRI, $135 \mathrm{ng} / \mathrm{l}$ to $601 \mathrm{ng} / \mathrm{l}$ for NT-proBNP, and $2.7 \mathrm{mg} / \mathrm{l}$ to $15.1 \mathrm{mg} / \mathrm{l}$ for CRP, respectively. †Optimal cut-off values were $\geqslant 2$ for RCRl, $\geqslant 301 \mathrm{ng} / \mathrm{l}$ for NT-proBNP, and $\geqslant 3.4 \mathrm{mg} / \mathrm{l}$ for CRP.

$\S$ Defined by at least two of three risk predictors are higher than cut-off values.

AMI, acute myocardial infarction; CV Death, primary cardiovascular death; PE, pulmonary oedema; PMCE, perioperative major cardiovascular event.

Figure 3 Receiver-operating characteristic (ROC) analysis of perioperative risk predictors. The predictive power of each combination of categorised risk predictor in an additive manner was investigated. Risk predictors were categorised according to the optimal cut-off levels derived from ROC analysis, which were 2 for RCRI, $301 \mathrm{ng} / \mathrm{l}$ for BNP and $3.4 \mathrm{mg} / \mathrm{l}$ for CRP. Areas under the curve (AUCs) with $95 \% \mathrm{Cl}$ are shown below each panel. ${ }^{*} p<0.05$ by Hanley and McNail methods. (A) ROC for PMCE. ${ }^{*} p<0.001$ for all, except RCRI + NT-proBNP vs RCRI + NT-proBNP + CRP $(p=0.001)$, and RCRI + CRP vs RCRI + NT-proBNP ( $p=0.010)$. (B) ROC for AMI. ${ }^{*} p<0.001$ for all, except RCRI + NTproBNP vs RCRI + NT-proBNP + CRP $(p=0.026), R C R I+C R P$ vs RCRI + NTproBNP + CRP $(p=0.002)$ and $R C R I+$ CRP vs RCRI + NT-proBNP ( $p=0.590)$. (C) ROC for pulmonary oedema. ${ }^{*} p<0.001$ for all, except RCRI + CRP vs $\mathrm{RCRI}+\mathrm{NT}-$ proBNP $(\mathrm{p}=0.004)$ and RCRI + NT-proBNP vs RCRI + NT-proBNP + $\operatorname{CRP}(p=0.001)$. (D) ROC for primary cardiovascular death. ${ }^{*} \mathrm{RCRI}$ vs CRP, $p=0.021 ;$ RCRI vs RCRI + NT-proBNP, $\mathrm{p}=0.012 ; \mathrm{RCRI}$ vs RCRI + NT-proBNP + CRP, $p=0.002 ; R C R I+C R P$ vs RCRI + NT-proBNP + CRP, $p=0.021$. AMI, acute myocardial infarction; CV death, primary cardiovascular death; $\mathrm{PE}$, pulmonary oedema; PMCE, perioperative major cardiovascular event.
A

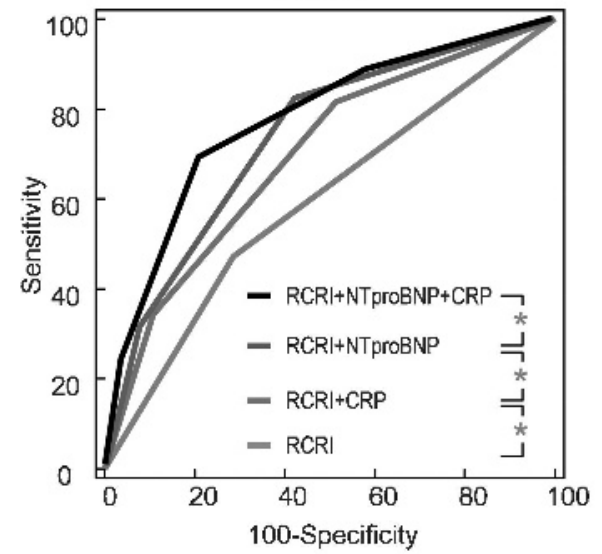

RCRI+NTproBNP+CRP $\quad 0.772 \pm 0.017[95 \% \mathrm{Cl} 0.752-0.790]$ RCRI+NTproBNP $\quad 0.735 \pm 0.018$ [95\% Cl 0.714-0.754] RCRI+CRP $0.694 \pm 0.019[95 \% \mathrm{Cl} 0.673-0.715]$ RCRI $\quad 0.592 \pm 0.019[95 \% \mathrm{Cl} 0.570-0.615]$

C

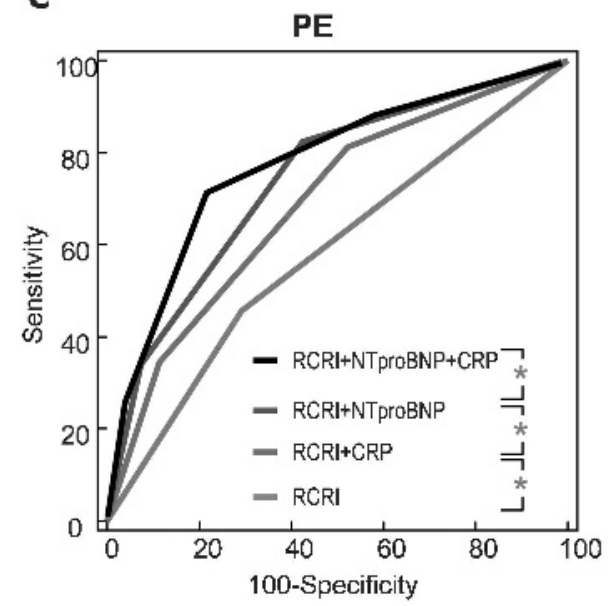

RCRI+NTproBNP+CRP $\quad 0.777 \pm 0.018[95 \% \mathrm{Cl} 0.758-0.796]$

RCRI+NTproBNP $\quad 0.738 \pm 0.019$ [ $95 \% \mathrm{Cl} 0.718-0.758$ ]

RCRI+CRP $0.689 \pm 0.020[95 \% \mathrm{Cl} 0.668-0.710]$

RCRI $\quad 0.582 \pm 0.020[95 \% \mathrm{Cl} 0.560-0.604]$
B

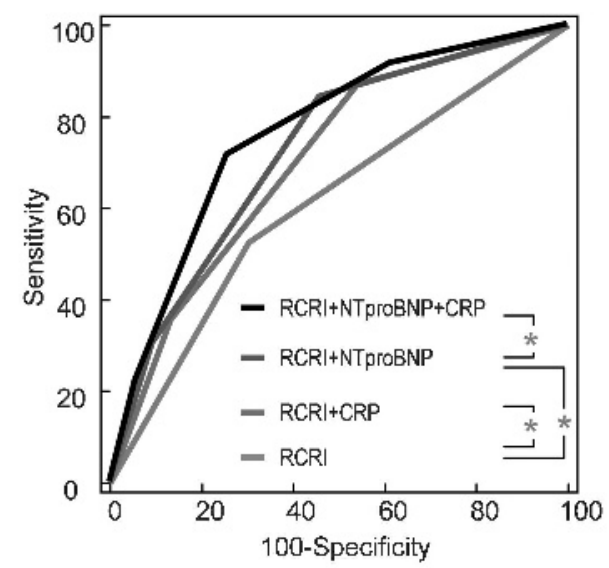

RCRI+NTproBNP+CRP $\quad 0.761 \pm 0.029$ [95\% Cl 0.741-0.780]

RCRI+NTproBNP $\quad 0.720 \pm 0.030[95 \% \mathrm{Cl} 0.700-0.740]$

RCRI+CRP $\quad 0.705 \pm 0.031[95 \% \mathrm{Cl} 0.685-0.726]$

RCRI $\quad 0.611 \pm 0.031$ [95\% Cl $0.589-0.633]$

D

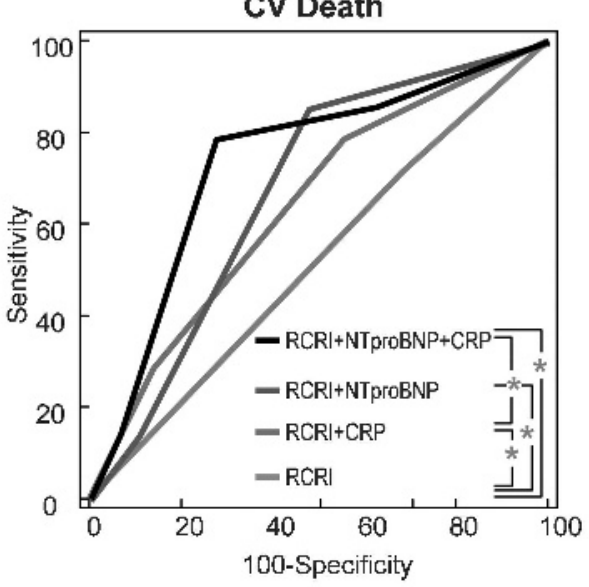

RCRI+NTproBNP+CRP $\quad 0.737 \pm 0.077$ [95\% Cl $0.717-0.757]$

RCRI+NTproBNP $\quad 0.678 \pm 0.080$ [95\% Cl $0.657-0.699]$ RCRI+CRP $\quad 0.639 \pm 0.080$ [95\% CI $0.617-0.660]$

RCRI $\quad 0.515 \pm 0.077[95 \% \mathrm{Cl} 0.492-0.537]$ 
was selected as the clinical risk predictor in our study. ${ }^{2-5}$ The results showed that a single cardiovascular biomarker, NTproBNP or CRP, is superior to clinical risk indices for the prediction of perioperative events. In addition, both NT-proBNP and CRP were significantly associated not only with all clinical events but also with all subsidiary events including AMI, pulmonary oedema and primary cardiovascular death, whereas RCRI was not associated with primary cardiovascular death. Furthermore, NT-proBNP and CRP were not only useful for risk prediction but also shown to improve the predictive power of clinical risk index. The addition of NT-proBNP and CRP to the clinical risk index increased the adjusted relative risk threefold.

\section{Role of NT-proBNP and CRP in the pathophysiology of perioperative cardiovascular events}

The pathophysiology of perioperative myocardial infarction has been explained by responses to perioperative surgical stress represented by a catecholamine surge with associated haemodynamic stress, systemic inflammation and hypercoagulability. ${ }^{18} 19$ High NT-proBNP was associated not only with a high risk of pulmonary oedema but also with AMI and primary cardiovascular death in our study. This could be explained by the release of NT-proBNP or B-natriuretic peptide (BNP) from subclinical ischaemic or injured myocardial tissue regardless of haemodynamic stress. ${ }^{12}{ }^{20}$ Our results strongly suggest that NTproBNP could be marker of myocardial ischaemia or generalised cardiac impairment in perioperative situations as well as nonsurgical situations. The predictive power of CRP was also better than the clinical risk index in this study. However, the high CRP levels did not predict clinical events beyond NT-proBNP. Previous population-based studies showed that the value of CRP in cardiovascular risk prediction was moderate or less than traditional risk factors. ${ }^{21} 22$ A recently published study showed that NT-proBNP was better than CRP for the prediction of sudden cardiac death. ${ }^{23}$ A relatively high CRP level in perioperative conditions might lead to a greater contribution of CRP to the risk prediction in our study.

\section{Limitations}

This study was performed at single centre. Only patients who had undergone formal preoperative cardiovascular consultation were included. However, given the strength of our results and wide variability in the predictive power of risk indices which highly depend on study population, it is unlikely that enrolment of more patients would have changed the main results of our study. ${ }^{1}$ Long-term follow-up after discharge was not performed, although most postoperative cardiovascular events are known to develop in early postoperative periods. ${ }^{24}{ }^{25}$ Exclusion of patients with renal dysfunction might have affected the predictive power of RCRI, which includes renal function evaluation. ${ }^{1326}$ Therefore, our results cannot be generalised to patients with renal dysfunction. The presence of preoperative infection or the use of antibiotics, which might affect the level of CRP or the severity of perioperative systemic inflammation, was not used as an exclusion criterion. The relation between infection and perioperative cardiovascular disease is little known and requires further investigation. The use of a preoperative $\beta$ blocker or statin was less than $20 \%$ and was not associated with clinical events in our study. The benefit of a $\beta$-blocker or statin is currently in debate even in high-risk patients, and large prospective trials are needed to confirm it..$^{27} 28$

In conclusion, our results demonstrate that high preoperative NT-proBNP and CRP levels are strong and independent predictors of perioperative cardiovascular events following non-cardiac surgery. Furthermore, the predictive power of the current clinical perioperative risk index could be strengthened significantly by the addition of these biomarkers. Evaluation of preoperative NT-proBNP and CRP is a practical, simple and reasonable strategy to improve perioperative risk prediction with minimal clinical burden and cost.

Funding: This study was supported by grants from the Samsung Medical Center Clinical Research Development Program and the In-Sung Foundation for Medical Research, Seoul, Korea.

Competing interests: None declared.

Provenance and peer review: Not commissioned; externally peer reviewed.

\section{REFERENCES}

1. Devereaux PJ, Goldman L, Cook DJ, et al. Perioperative cardiac events in patients undergoing noncardiac surgery: a review of the magnitude of the problem, the pathophysiology of the events and methods to estimate and communicate risk. CMAJ 2005;173:627-34.

2. Goldman L, Caldera DL, Nussbaum SR, et al. Multifactorial index of cardiac risk in noncardiac surgical procedures. N Engl J Med 1977;297:845-50.

3. Detsky AS, Abrams HB, Forbath $\mathrm{N}$, et al. Cardiac assessment for patients undergoing noncardiac surgery. A multifactorial clinical risk index. Arch Intern Med 1986;146:2131-4.

4. Lee TH, Marcantonio ER, Mangione CM, et al. Derivation and prospective validation of a simple index for prediction of cardiac risk of major noncardiac surgery. Circulation 1999;100:1043-9.

5. Kertai MD, Boersma E, Klein J, et al. Optimizing the prediction of perioperative mortality in vascular surgery by using a customized probability model. Arch Intern Med 2005:165:898-904.

6. Poldermans D, Bax JJ, Schouten 0, et al. Should major vascular surgery be delayed because of preoperative cardiac testing in intermediate-risk patients receiving beta-blocker therapy with tight heart rate control? J Am Coll Cardiol 2006;48:964-9

7. Falcone RA, Nass $\mathrm{C}$, Jermyn $\mathrm{R}$, et al. The value of preoperative pharmacologic stress testing before vascular surgery using ACC/AHA guidelines: a prospective, randomized trial. J Cardiothorac Vasc Anesth 2003;17:694-8.

8. Fleisher LA, Beckman JA, Brown KA, et al. ACC/AHA 2007 guidelines on perioperative cardiovascular evaluation and care for noncardiac surgery: a report of the American College of Cardiology/American Heart Association Task Force on Practice Guidelines (Writing Committee to Revise the 2002 Guidelines on Perioperative Cardiovascular Evaluation for Noncardiac Surgery) developed in collaboration with the American Society of Echocardiography, American Society of Nuclear Cardiology, Heart Rhythm Society, Society of Cardiovascular Anesthesiologists, Society for Cardiovascular Angiography and Interventions, Society for Vascular Medicine and Biology, and Society for Vascular Surgery. J Am Coll Cardiol 2007:50:e159-241.

9. Kistorp C, Raymond I, Pedersen F, et al. N-terminal pro-brain natriuretic peptide, Creactive protein, and urinary albumin levels as predictors of mortality and cardiovascular events in older adults. JAMA 2005;293:1609-16

10. Zethelius B, Berglund L, Sundstrom J, et al. Use of multiple biomarkers to improve the prediction of death from cardiovascular causes. $N$ Engl J Med 2008;358:2107-16.

11. Timpson NJ, Lawlor DA, Harbord RM, et al. C-reactive protein and its role in metabolic syndrome: mendelian randomisation study. Lancet 2005;366:1954-9.

12. Morrow DA, de Lemos JA, Blazing MA, et al. Prognostic value of serial B-type natriuretic peptide testing during follow-up of patients with unstable coronary artery disease. JAMA 2005;294:2866-71.

13. Goei D, Schouten O, Boersma E, et al. Influence of renal function on the usefulness of $\mathrm{N}$-terminal pro-B-type natriuretic peptide as a prognostic cardiac risk marker in patients undergoing noncardiac vascular surgery. Am J Cardiol 2008;101:122-6.

14. Laine C, Williams SV, Wilson JF. In the clinic. Preoperative evaluation. Ann Intern Med 2009;151:ITC1-16.

15. Stephan C, Wesseling S, Schink T, et al. Comparison of eight computer programs for receiver-operating characteristic analysis. Clin Chem 2003;49:433-9.

16. Zhang J, Yu KF. What's the relative risk? A method of correcting the odds ratio in cohort studies of common outcomes. JAMA 1998;280:1690-1.

17. Hahn JY, Gwon HC, Park SW, et al. The clinical features of transient left ventricular nonapical ballooning syndrome: comparison with apical ballooning syndrome. Am Heart J 2007:154:1166-73.

18. Poldermans D, Hoeks SE, Feringa HH. Pre-operative risk assessment and risk reduction before surgery. J Am Coll Cardiol 2008;51:1913-24.

19. Schouten 0, Bax JJ, Poldermans D. Preoperative cardiac risk assessment in vascular surgery patients: seeing beyond the perioperative period. Eur Heart $J$ 2008;29:283-4.

20. Sabatine MS, Morrow DA, de Lemos JA, et al. Acute changes in circulating natriuretic peptide levels in relation to myocardial ischemia. J Am Coll Cardiol 2004:44:1988-95 
21. Van der Meer IM, de Maat MP, Kiliaan AJ, et al. The value of C-reactive protein in cardiovascular risk prediction: the Rotterdam Study. Arch Intern Med 2003;163:1323-8.

22. Pradhan AD, Manson JE, Rossouw JE, et al. Inflammatory biomarkers, hormone replacement therapy, and incident coronary heart disease: prospective analysis from the Women's Health Initiative observational study. JAMA 2002;288:980-7.

23. Korngold EC, Januzzi JL Jr, Gantzer ML, et al. Amino-terminal pro-B-type natriuretic peptide and high-sensitivity C-reactive protein as predictors of sudden cardiac death among women. Circulation 2009;119:2868-76.

24. McFalls EO, Ward HB, Moritz TE, et al. Predictors and outcomes of a perioperative myocardial infarction following elective vascular surgery in patients with documented coronary artery disease: results of the CARP trial. Eur Heart J 2008;29:394-401.
25. Owens CD, Ridker PM, Belkin M, et al. Elevated C-reactive protein levels are associated with postoperative events in patients undergoing lower extremity vein bypass surgery. J Vasc Surg 2007;45:2-9; discussion 9.

26. McCullough PA, Duc P, Omland T, et al. B-type natriuretic peptide and renal function in the diagnosis of heart failure: an analysis from the Breathing Not Properly Multinational Study. Am J Kidney Dis 2003;41:571-9.

27. Devereaux PJ, Yang H, Yusuf S, et al. Effects of extended-release metoprolol succinate in patients undergoing non-cardiac surgery (POISE trial): a randomised controlled trial. Lancet 2008;371:1839-47.

28. Bangalore S, Wetterslev J, Pranesh S, et al. Perioperative beta blockers in patients having non-cardiac surgery: a meta-analysis. Lancet 2008;372:1962-76. 